\title{
Revolutionizing Healthcare with IoT and Cognitive, Cloud-based Telemedicine
}

\author{
Ábel Garai, István Péntek, Attila Adamkó \\ University of Debrecen, Faculty of Information Technology, Kassai út 26, 4028 \\ Debrecen, Hungary, garai.abel@inf.unideb.hu, pentek.istvan@inf.unideb.hu, \\ adamko.attila@inf.unideb.hu
}

\begin{abstract}
Telemedicine instruments and e-Health mobile wearable devices are designed to enhance patients' quality of life. The adequate man-and-machine cognitive ecosystem is the missing link for that in healthcare. This research program is dedicated to deliver the suitable solution. This research's goal is the establishment of adaptive informatics framework for telemedicine. This is achieved through the deployed open telemedicine interoperability hub-system. The presented inter-cognitive sensor-sharing system solution augments the healthcare ecosystem through extended interconnection among the telemedicine, IoT e-Health and hospital information system domains. The general purpose of this experiment is building an augmented, adaptive, cognitive and also universal healthcare information technology ecosphere. This study structures the actual questions and answers regarding the missing links and gaps between the emerging Sensor Hub technology and the traditional hospital information systems. The Internet-of-Things space penetrated the personal and industrial environments. The e-Health smart devices are neither widely accepted nor deployed in the ordinary healthcare service. This paper reviews the major technological burdens and proposes necessary actions for enhancing the healthcare service level with Sensor Hub and Internet-of-Things technologies. Hereby we report the studies on varying simplex, duplex, full-duplex, data package- and file-based information technology modalities. We establish with that stable system interconnection among clinical instruments, healthcare systems and eHealth smart devices. Our research is based on the trilateral cooperation comprising the University of Debrecen Department of Information Technology, Semmelweis University Second Paediatric Clinic and T-Systems Healthcare Competence Center Central and Eastern Europe.
\end{abstract}

Keywords: Cognitive healthcare; telemedicine; telecare; e-health; IoT; sensor hub; hybrid cloud; healthcare IT

\section{Introduction}

Today, the information revolution affects all areas of life as people use continuously electronic devices on a regular basis. These equipments assist their users to simplify their everyday life, for example avoiding traffic jams or tracking 
their fitness activity. These gadgets hold various sensors and built-in interfaces to share the collected data with external tools and systems. Our aim is to let the produced data used in as many ways as possible.

The most common and widely used hardware component in smart devices is the global positioning system (GPS) sensor. Most of the mobile phones have GPS capability and host multiple mobile applications using this sensor. Navigation applications collect and analyse the collected GPS coordinates and share these with further applications running on servers. These server applications process the received coordinates and calculate the best route to reach the destination or help the user to avoid traffic jams. Using navigation applications became part of our everyday life. Another commonly used device group consists of the fitness activity trackers. These collect health-related data as long as their users wear them [Figure 1]. Usually, users wear the fitness trackers during fitness activities like cycling, running, swimming, etc. These trackers are capable of collecting heart rate values, temperature, air pressure values or even more health-related data.

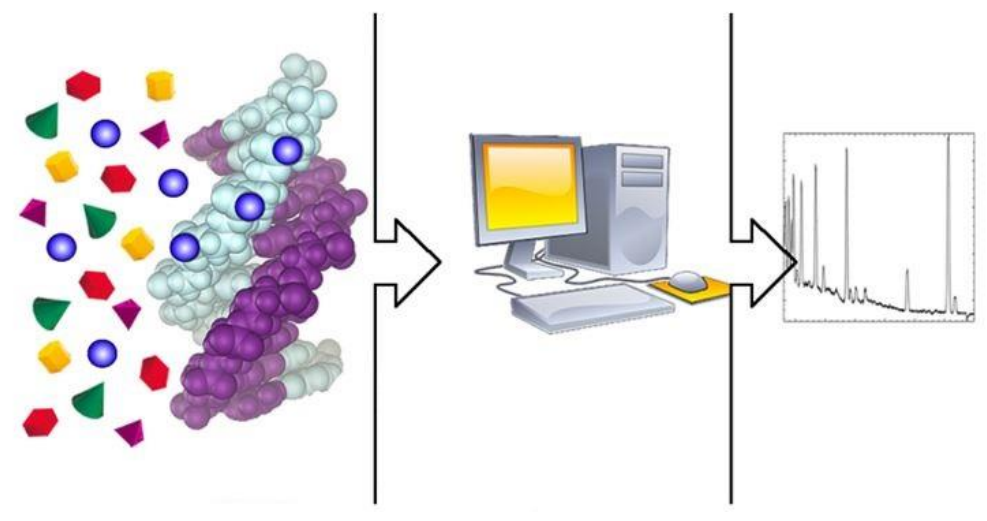

Figure 1

Biosensory data processing with healthcare IoT devices

Multiple applications can process these measurement values, but most of them are only simple client applications on the user's device. Client applications are working locally on their host mobile device (smartphone) and usually do not share the measurement results with external systems. If an application shares the measurement values, mostly it does it just to archive them. The hospital information systems unfortunately cannot work with large amounts of healthrelated measurement values: they cannot access them since they usually remain in separated, closed systems.

The doctor or specialist could use the tracked data during a medical examination if it is immediately accessible to him. The measurement values are helpful when the patient requires treatment, and the doctor needs to examine different parameters to make a justified decision [1]. For certain medical examination rely on measured 
heart rate values: these values can be extracted from fitness trackers or wearable devices with our solution. Sensor-based wearable devices are producing sufficient volume of health-related data every day [2]. Our article focuses on sensor data and describes our developed hub system between the internet of things (IoT) devices and health-related systems. In particular, the actual phase of our research concentrates on privacy and the credibility of the measurement chain.

\section{Cognitive Telemedicine}

This paper derives and adapts the principles of cognitive info-communications [3] to our described scientific research. The application of the given synergies leads to the foundation of the cognitive telemedicine. The inter-cognitive sensor-bridging communication is the specific area, where our research and the cognitive info communication are interlocked [4]. The human-machine interaction has been researched since 1976 [5]. The Cognitive Infocommunication presents the next significant milestone concerning the human-ICT interconnection. Our paper augments this scientific area with the practical e-Health implementation. Therefore, our research delivers the operational information technology realization for the next generation human-machine interaction for the e-Health. In our research the human patients are interconnected through bio-sensory e-Health devices to the international information technology landscapes and also to further human actors.

Our previously proposed private cloud architecture [15] gives room for intracognitive sensor-bridging and inter-cognitive sensor-sharing communications. This is the suitable category of Cognitive Infocommunications for the enhanced telemedicine systems allowing doctors to assess remotely patients' physiological, psychological and neural state. The cloud architecture provides the link between the cloud architectural solution for telemedicine systems and the Cognitive Infocommunications: patient information is directed to the doctor using the telemedicine cognitive subsystem, while the data is captured by medical sensors. As telesurgery systems gain ground, the drafted cloud computing architecture links the human doctor with the remote surgery machine: it concludes an intracognitive sensor-sharing cognitive info communication. [5]

Our research plays a significant role in the enhancement of the Human and Biointerfaces chapter of the Cognitive Infocommunication [6] discipline. There were already significant scientific achievements published in this area, for example: "The significance of cognitive info-communications in developing assistive technologies for people with non-standard cognitive characteristics: CogInfoCom for people with nonstandard cognitive characteristics" [7]. Our presented paper's secondary area within the Cognitive Infocommunications is the "Human factors, E-health, and People with Specific Needs". Two previous major publications 
within this topic are „Cognitive Infocommunication for Monitoring and Improving Well-being of People" [8] and "Cognitive workload classification using cardiovascular measures and dynamic features" [9].

Telemedicine involves the distribution of health-related services and information via electronic information and telecommunication technologies [10]. There is no single definition for telemedicine systems. Some definitions include all aspects of medical care including also preventive health care. Others use telemedicine and telehealth interchangeably. Therefore, our definition of telemedicine is the use of information technology and telecommunication to provide clinical health care from a distance. It is used to overcome the distance and to improve access to health care services where it is not consistently available.
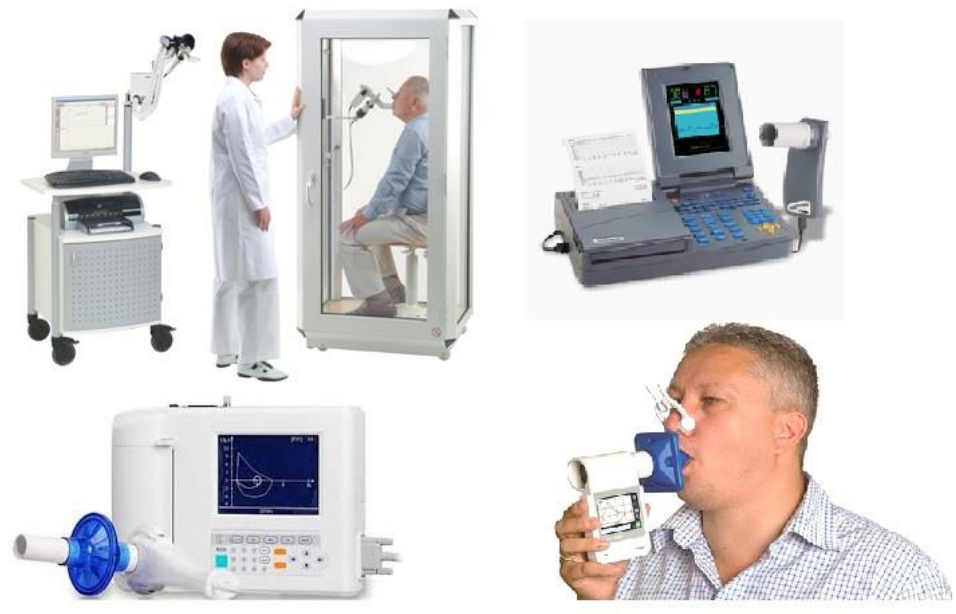

Figure 2

Spirometer devices for telemedicine

Telemedicine systems are also used to provide better outcomes in critical care and emergency solutions [11]. Telemedicine depends on the $20^{\text {th }}$ Century telecommunication and information technologies [Figure 2]. These technologies ensure the communication between patients and medical staff; and they help to transmit health-related data and images reliably from one site to another. The first form of telemedicine was relying on simple telephone connections. Later, the advanced medical diagnostic methods were supported by client-server applications working with additional telemedicine devices to support in-home medical examinations.

Telemedicine allows medical contact and healthcare services from distance. It has many forms: supporting advice-giving, making health-related reminders, education, remote admissions, remote monitoring and healthcare system integration. Telemedicine should make learning, supervision and health data 
management simpler even when the required expert and the patient are far away from each other. With telemedicine, the patient and the expert can make clinical discussion over video conference.

Telemedicine does not purely consist of technology and devices, but it has a determining social aspect. Telemedicine improves access to health care services that would often not be consistently available in distant communities [12] [13]. Patients need to transmit important and sensitive messages, personal health records through a publicly available network connection. It will happen only if people trust the telemedicine systems and the underlying technologies.

The most important telemedicine or telehealth feature regarding our presented article is the patient remote monitoring or home monitoring. This feature allows the expert to follow the patient's health-related measurement values. While the patient is using a wearable device or devices the telemedicine system can read the data measured by the device. To achieve real-time health monitoring, the expert and the patient must have reliable, uninterrupted internet connection, trusted wearable healthcare device or devices, and a software to evaluate the measurement data.

\subsection{Sensor-based Adaptive e-health Systems}

Telemedicine and information technology allow to create systems that collect, transform and transmit the data measured by the users' wearable devices. The most people have one or more wearable devices which can record health-related data, these are typically fitness trackers. Fitness trackers can follow multiple health-related information during a fitness activity. The most fitness tracker can measure the current heart rate value, air pressure, calories, sleep quality, altitude, distance, and steps. The most tracker contains GPS locator, it could be useful in case of urgent medical cases.

The trackers can collect multiple information about the wearer and they could send them to a telemedicine system that can process the received data and send notification or alert if it is necessary [14]. These telemedicine systems could be integrated to any other health-related systems. Hospital information systems or other medical systems should use the information received from telemedicine systems. The system vendors are responsible for integrating external telemedicine systems and using the publicly available and free health-related data. This large amount of publicly available data is suitable for making a more accurate medical decision and creating real-time health monitoring systems [Figure 3]. 


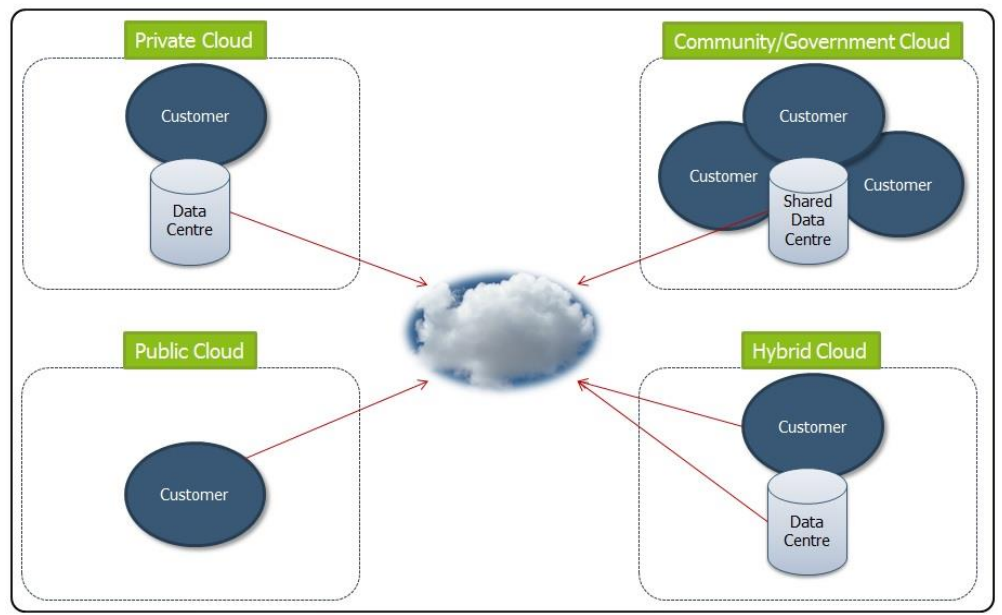

Figure 3

Cloud architecture types for healthcare services

The most tracker has multiple sensors that capable to record different healthrelated data, e.g.: heart rate value or calories. Beyond the fitness trackers, there are several further devices that capable to record health-related data. Smart scale records the users' weight as often as the users' uses that. Blood glucose meter for home use records the users' current blood glucose level. Smart blood pressure meter records the current blood pressure and has multiple interfaces to transfer the result to external device or system. In a modern home can be found multiple devices that can collect health-related measurement values: that could be useful during a medical examination [15].

If the patient trusts the external system and the medical expert handles the measurement values with reservations, the sensor based telemedicine systems could be a useful part of the medical- or the hospital information systems.

\subsection{Implementing IoT in the Healthcare Supply Chain}

Internet of things is the network of devices embedded with multiple sensors and capability of network connectivity enabling these objects to connect to other devices or systems sharing information with them. An IoT object can be sensed or controlled remotely across the existing network infrastructure [16]. It gives the opportunity for direct integration of these objects into external systems. The "things" in the IoT expression can refer to any device equipped with sensors and holding an active network connection [17]. Today, most types of these devices have sensory and network capability. There are many application areas for the internet of things as shown in Figure 4: 


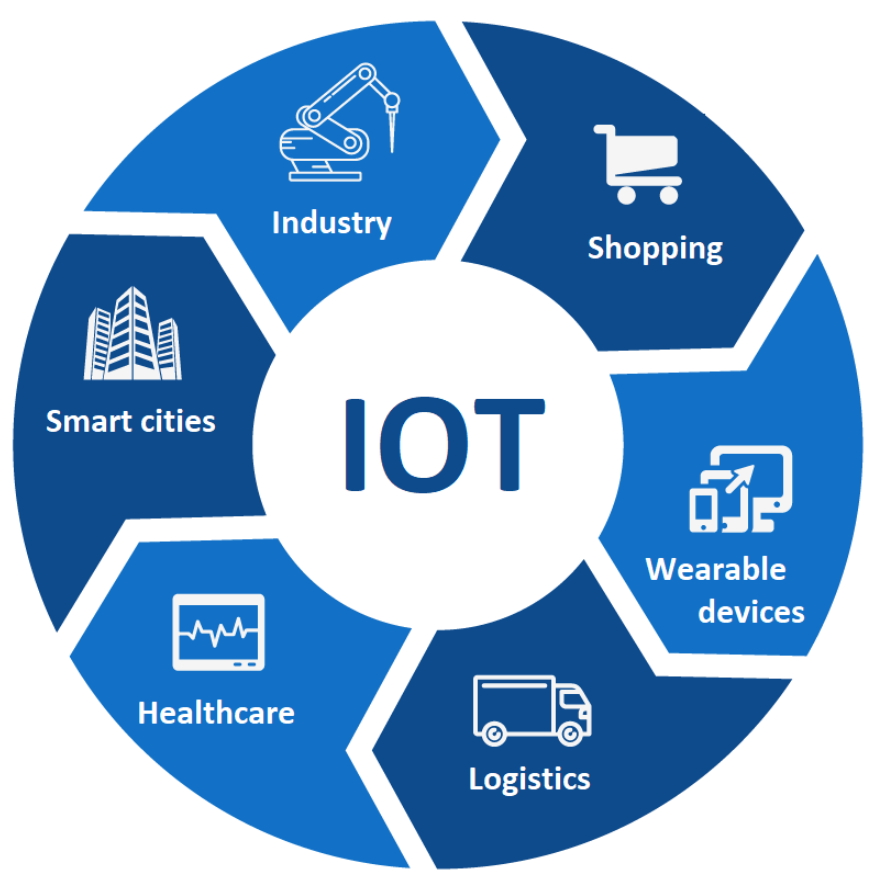

Figure 4

Application areas for IoT

As it was described earlier in this article most people have multiple devices to record health-related measurement data or non-health-related measurement values. If these devices have one or more interfaces to connect external systems or other devices the measured values can be forwarded for further processing in industrial healthcare systems. This ability makes the data aggregation reasonable from a simple wearable sensory device into a commercial telemedicine system.

\subsection{Open Telemedicine Hub-Software}

Open telemedicine interoperability (OTI) hub is a complex application based on internet of things devices. It provides a set of publicly available application programming interfaces (API). OTI hub allows IoT devices to share health-related measurements with other systems. Through the open API, the health-related systems use the provided health records. The OTI hub provides the information in multiple formats. To serve the requests from the hospital information system the OTI hub uses HL7 formats. This format is widely accepted in the international healthcare domain. Figure 5 represents the relevant healthcare data sources and consumers: 


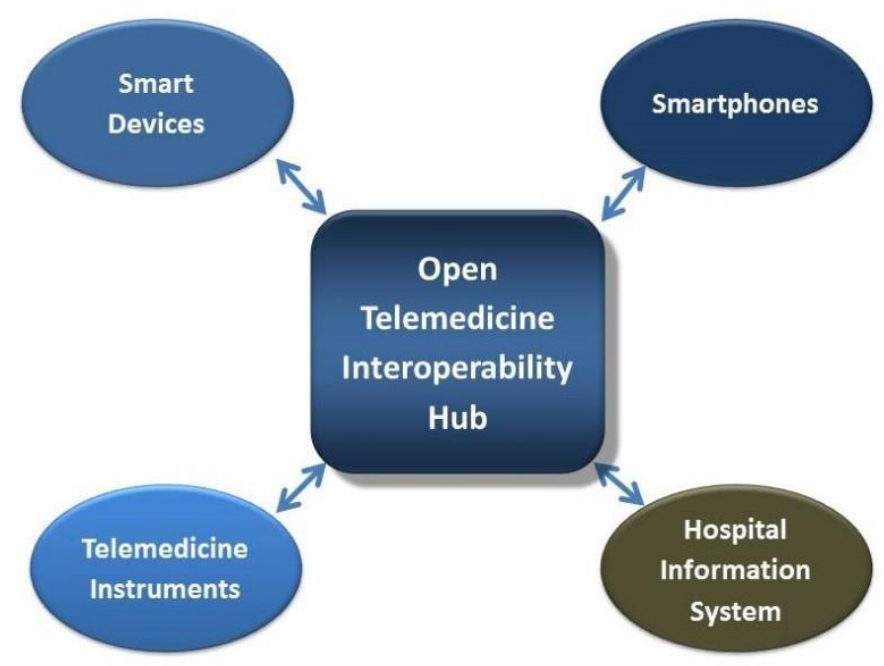

Figure 5

Open telemedicine interoperability hub-software's data-link diagram

The OTI hub collects the measurement values from the health-related devices and cleans the received data from the measurement errors. The error-free measurement values are stored for further use. The integrated healthcare systems can create parameter-based customized reports. The retrieved information is used during medical examinations and for disease-prediction [18]. The telemedicine environment was adjusted to meet the requirements of the statistical evaluation of the captured bio-sensory data [19].

The OTI hub provides useful real-time health monitoring. In our case, the OTI hub works as a cross-functional channel between the smart end-devices and industrial healthcare systems. The OTI hub itself does not make medical decisions: it acts as a proxy transforming and transmitting the measurement values to the integrated systems in the requested format and structure. Then, the external systems issue reports, notifications and alerts based on the received values. Figure 6 shows the OTI hub's reference architecture: 


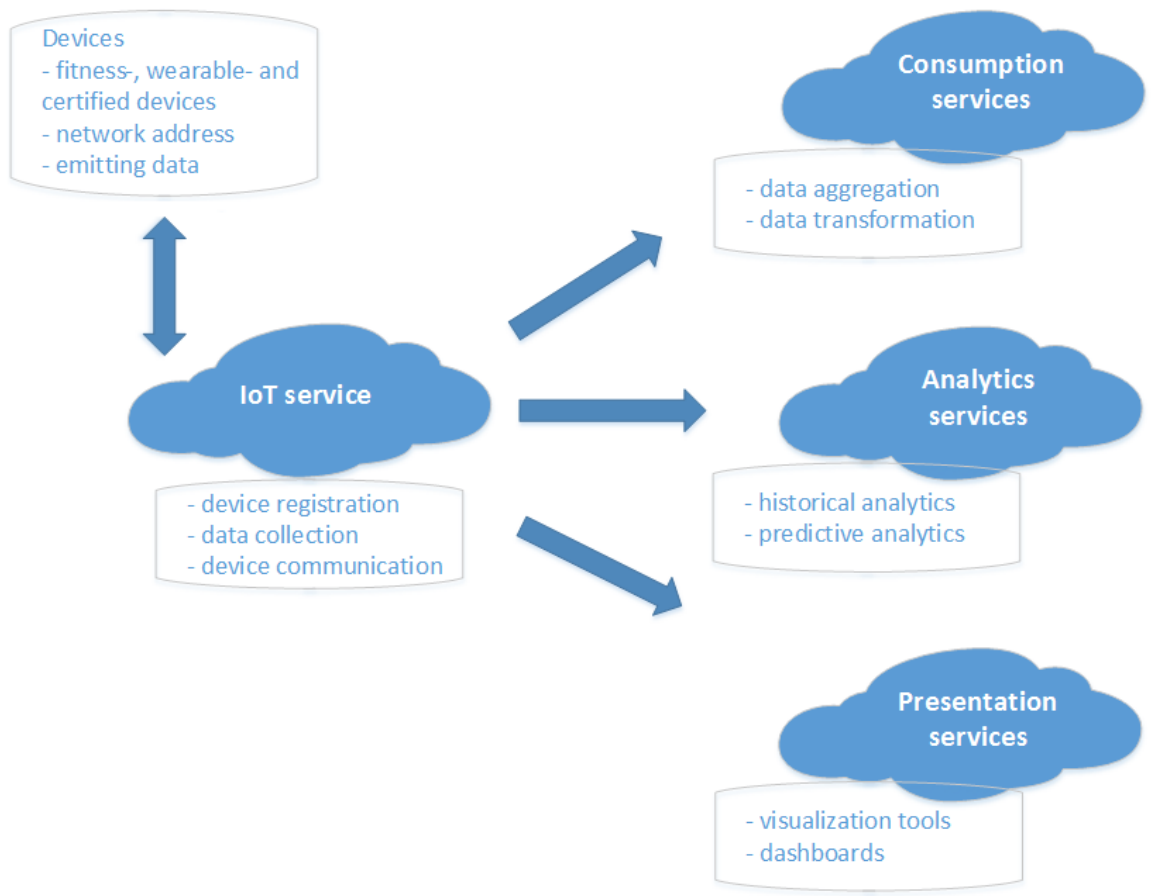

Figure 6

Open telemedicine interoperability hub-software's reference architecture

The OTI hub has the following common IoT services:

- Consumption services: these services are responsible for data aggregation and data transformation. They prepare the captured measurement values for archiving them. During the transformation phase, the measurement errors are removed.

- Analytics services: it is responsible for creating analytics on the historical data-flow and making predictions. The prediction function is applied during medical examinations. The analytics services are using machine learning algorithms relying on pattern analysis.

- Presentation services: these services visualize the received measurement values to the users. Visualization is real-time, and it is based on current values.

\subsection{Research Methodology and Software Technology}

Clinical systems interoperability reaches beyond plain data-exchange: it constitutes interoperability at technical, semantic and at the process level. In the empirical model of the research the OSI model (ISO/IEC 7498-1:1994 [20]) is mapped against the aforementioned interoperability levels. Therefore, these three interoperability modalities are interpreted also at the corresponding information 
technology abstraction layer. Technical and semantic interoperability is targeted within the presented research. Among the technical interoperability modalities instead of the TCP/IP, the file-based interface connection has been elected: this option offered significantly more flexibility during the research, as shown in Figure 7:

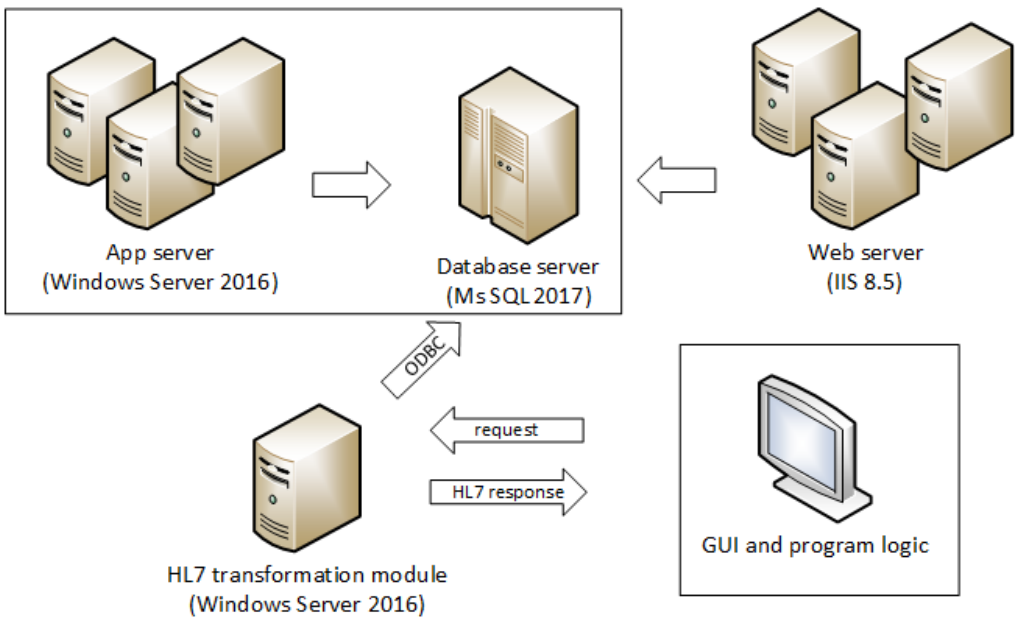

Figure 7

Open telemedicine interoperability hub-software's architecture

The following instruments have been selected and allocated to the research program: Spirometer PDD-301/shm as clinical telemedicine instrument, Microsoft Band I and Microsoft Band II smart wristband as eHealth sensory devices, Nokia Lumia 930 smartphone (Windows 10 Mobile operating system), Dell Latitude E6520 (Windows 1032 bit operating system, i5-2520M chipset, 4 GB RAM and 256 GB HDD) primary laptop, Dell Latitude E6220 (Windows 764 bit operating system, i5-2520M chipset, 4 GB RAM, 128 GB SDD) secondary laptop, three Lenovo MIIX 300-10IBY tablets and an ACER SWITCH SW3-013-12CD tablet. Each tablet is equipped with 10,1 display (WXGA and HD IPS), 2 GB memory, 64 GB internal storage and Windows 10 operating system. All laptops and tablets fit the 802.11g WLAN and Bluetooth 4.0 standards. The spirometer is USBenabled. The selected smart wristbands are manufactured with built-in- Bluetooth 4.0 communication chipsets. Each instrument of the lab equipment package has been individually tested prior to the experiment.

A specific communication link over was established between the smartphone and the bio-sensory healthcare IoT device, as shown in Figure 8. 


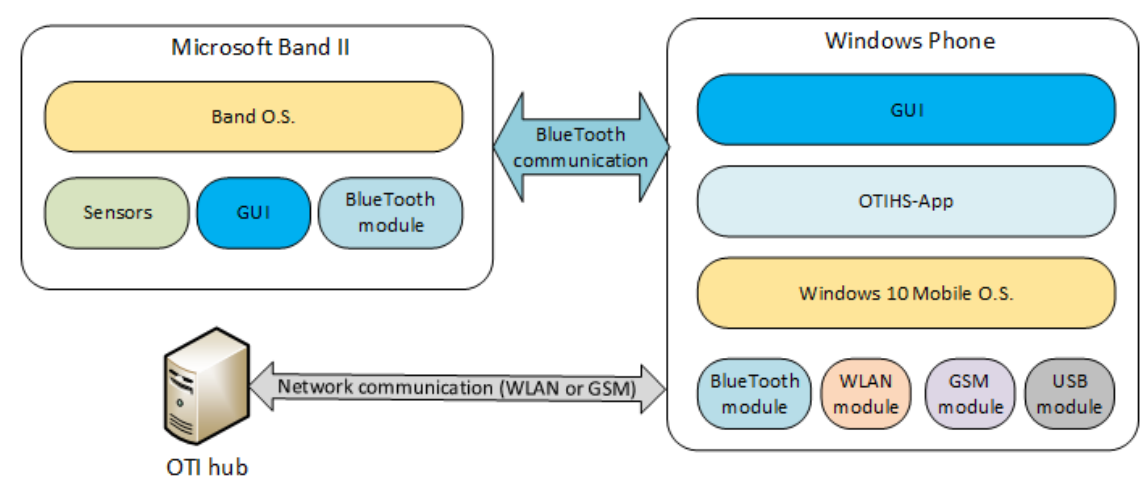

Figure 8

Communication link for OTI-HS app and bio-sensory device

A specific private cloud was established for the research. This ran on stand-alone x86-64 architecture equipped with Intel i5 processor, 256GB SSD, and 4 GB RAM. The operating system for the private Cloud is Red Hat Enterprise Linux 7.0 3.10.0229, the virtualization is provided by VMware Workstation v6.5.0 and the relational database management system is supplied by MySQL v5.6. The cloudbased version of the hospital information test system runs in a commercial private cloud (Telekom Cloud). The Open Telemedicine Interoperability Hub data transmission module is embedded in a commercial public cloud (Microsoft Azure).

The HIS runs on J2EE WebSphere Application server V6, relying upon Oracle RDBMS $10 \mathrm{gR} 2$ and Progress V10 OpenEdge RDBMS. The HIS is hosted on Unix operating system. Floating licenses were made available for reaching the online, cloud-based edition of the selected HIS through the research tablets. The Open Telemedicine Interoperability Hub development environment consisted of the Universal Windows Application Development Tools (1.4.1), Windows 10 Software Development Kit 10.0.25431.01 Update 3 and Microsoft .NET Framework Version 4.6.01038. The OTI-Hub internal database was developed by SQL Server Data Tools 14.0.60519.0. The OTI-Hub App was developed with Visual Studio Tools for Universal Windows Apps 14.0.25527.01. The OTI-Hub middleware was settled in Microsoft Azure Mobile Services Tools 1.4. Red Hat Enterprise Linux 7.0 3.10.0-229 provided the operating system for the private cloud established specifically for the research.

The spirometry desktop program has been installed on a standalone Dell Latitude E6520 laptop equipped with Windows 10 operating system. The spirometer has been calibrated by the manufacturer for the research. Forced vital capacity spirometry test has been undertaken with a healthy individual. Having the test results stored in the spirometry desktop software, the HL7 v2.3.1 interface file has been exported. This interface file has been processed by the cloud-based OTIHub. The OTI-Hub appended the spirometry information with the previously 
transformed cardio body sensor information captured by the L18 Smart Bluetooth Wristband. The generated HL7 interface file is imported after parameterization into the factory acceptance test instance of the MedSol hospital information system. Both the imported spirometry and cardio test results are retrieved and displayed in the patient report query of the hospital information system. The information technology results are validated by the Department of Information Technology, University of Debrecen and by T-Systems Healthcare Competence Center Central and Eastern Europe. The clinical results are validated by the Semmelweis University 2nd Department of Paediatrics.

The implemented system is a distributed, cloud-based and scalable. In case of load increase, the system can be scaled up by the automatic allocation of new resources into the OTI-Hub cluster.

\subsection{Research Methodology and Software Technology}

The OTI-Hub was interconnected to the mirrored HIS industry test system. The test plan included individual, cluster and integration tests. The individual tests concerned the single research software environment element: the receiver, transformational, storage, interpreter and integrational module of the OTI-Hub. The cluster tests focused both on the eHealth smart device and on the telemedicine instrument thread of the OTI-Hub.

The clinical spirometer emits elementary data. However, the smart wearable eHealth device produces continuous time-series. Therefore, a cluster test was carried out. During this cluster test, primary data both from the spirometer and from the wearable eHealth device was successfully processed. The integration test provided the overall quality assurance for the OTI-Hub. The telemedicine instrument and the eHealth smart device measured real bio-sensory signals of anonymized individuals and sent it to the OTI-Hub.

The OTI-Hub interpreted, saved, transformed and sent these data to the mirrored HIS industry test system. The allocated tablets were used to load the Cloud-based HIS graphical user interface. The tablets were connected via dedicated WLAN to the HIS industry test system. The results were validated through the GUI on the tablets by clinical professionals. However, the OTI-Hub module, which is responsible for the eHealth wearable device signals interception, proved to be unstable due to the regular mandatory operational system upgrade.

A separate load test was performed regarding the automatic cloud architecture scaling. For this validation, exponentially increasing number of parallel input was delivered to the dedicated cloud system. This test was successful as the virtual cloud infrastructure scaled up automatically to process the significantly increased workload. The load test was started with five compute-optimized virtual machines. These virtual machines were predefined with the following parameters: 16 virtual CPU cores, 32 GB allocated RAM and 256 GB allocated disk space. 
These tests simulated up to 100000 concurrent wearable eHealth device data flows and up to 10000 simultaneous simplified medical information system data flows. During these load tests, the virtual-wearable eHealth devices sent the test measurement values to the OTI-Hub. It processed, transformed and transmitted the captured measurement values into the simulated simplified medical information systems. The load test was successful, as the system successfully transmitted the previously specified number of transactions. A daily total 8500 000000 simulated heart rate transaction volume was processed without error during the load test.

\section{Modernizing Medical Solutions with IoT}

The modern medical solutions are required to apply the latest medical standards and to continuously follow the constantly changing laws and regulations. These circumstances and prerequisites are hard to fulfill by the medical contributors and vendors. Even market-leading medical solution can hardly keep pace with the always changing environment, and only a portion of them can integrate IoT capability [21] [22]. This article does not deal with the ruling healthcare laws and regulations, but it focuses on the data privacy and security challenges regarding IoT integration into the healthcare supply chain.

\subsection{Personal Assistant Roll-Out}

By collecting sensor data, we can continue our proposal with a new and interesting feature which forms a personal assistant for its users by analyzing data sets. Without analytics, our solution is only a half one. Analytics could drive our application and provide value-added services.

We can easily imagine several situations where the combination of different sources could result in interesting facts. While we are periodically measuring our heart rate by our smart bracelet we can correlate it with the user's calendar and GPS position to derive new facts, e.g. when we are on a business meeting its normal that our pulse could be over the normal values. It means, our system will not fire an alert when it detects some kind of abnormality in the measured values. Our system could be extended to learn these conditions, like the above average pulse on business meetings or on take-outs.

Naturally, the sources are endless. We can easily find smart scales, smart watches, smart blood pressure and smart blood glucose meters to collect not just the location, GPS coordinates or pulse from the users. The strength of our extensible architecture and data integrations is the possibility to derive new and useful information for our users. Like a recommendation and monitoring system which 
could share its data using a common format - in our case its HL7 with other medical application or Hospital Information Systems. We have made a short investigation about the used interchange formats in our national hospitals and that showed us to apply HL7 for export operations from our Med-i-Hub.

Based on the original example, using the calendar and location data we can find favorite places and events: so if it is connected to an Event management system, we can get event recommendations. Naturally, this is not the primary goal of our research just a use-case to expose the possibilities. It will much easier to derive information about places where users are feeling relaxed. Our assistant could learn from the location, event and pulse triplet: based on them recommendations could be provided when detecting abnormal user conditions.

These examples are highlighting that the Analytical module is playing a very important role in our research and the derived value-added services are forming the base for a visionary Personal Assistant application. Naturally, we need to find solutions for storing and analyzing this huge amount of data but the previously mentioned scalable architecture is full-filling these requirements.

\subsection{Solving the Data Privacy Issues for Telemedicine}

Available medical solutions are putting emphasis on data security and data privacy [23] [24]. It is critical to handle the user's data prudently in a secure manner. It is common to grant security using secure channels during the communication between the OTI hub and the end-users' devices; and also between the OTI hub and the industrial, integrated medical system.

There are various legal prerequisites and regulations in force to protect personal data in different geographical regions. However, health-related data protection rules are even stricter than common personal data protection rules.

The OTI hub communicates through secure channels with external environments and healthcare devices. The recorded bio-sensory data is handled according to predefined user's rules. The data owner classifies the recorded data. External systems can access the health-related data marked with the flag 'accessible' through the OTI hub. The data owner defines multiple rules for data accessibility. The medical systems can identify the user with the medical identifier. This identifier is issued by the users' healthcare institution.

The identifier is the key connecting the OTI hub user-ID with the examined patient in the medical system. The OTI hub does not require any other identification information from the external medical system, as it works as a data provider. In this sense the OTI hub does not handle sensitive personal information, therefore, it meets the required privacy and security level. 
The second method is when the OTI hub acts as a data consumer. In this case, the health-related data produced by the IoT devices are forwarded to the hub system. This forwarded health record holds sensitive personal information. Therefore, the OTI hub system handles these measurement values as sensitive personal information and these are handled according to the predefined user's accessibility rules.

\subsection{Safety and Security for Medical IoT Equipment}

The IoT revolution's biggest challenge is data security [25]. IoT devices sense multiple types of data and they share with external systems. Each type of captured data should have its own security level. There is also non-sensitive information transmitted by IoT devices, e.g. temperature or humidity. This kind of information does not need to be handled protected. However, another type of information, like GPS coordinates are sensitive. IoT devices do have generally applicable data security features. They use multiple types of networks where different security levels are required. The general rule is, that the aggregator system is always responsible for ensuring the security of the received data [26].

The second data security challenge is trust. The external systems must use the received information as reliable data received from a reliable source [27] [28]. Therefore, the consumer services use authentication and authorization. The most important security challenges are shown in Figure 9:

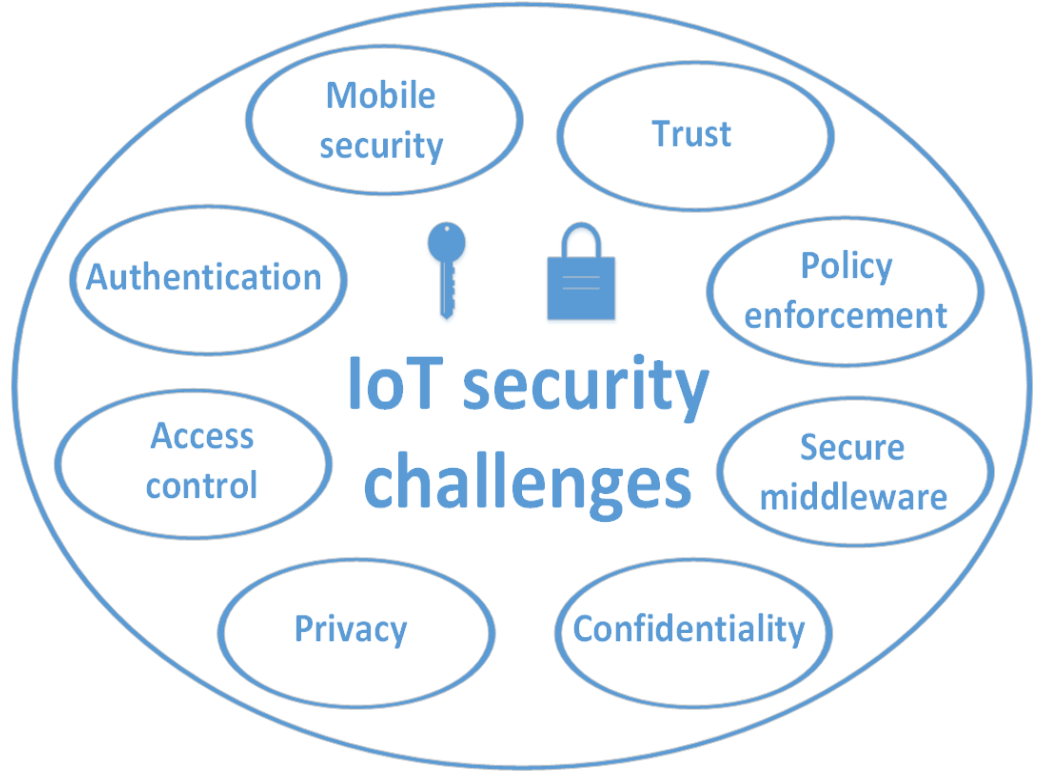

Figure 9

IoT security risks classification for e-health 


\subsection{Data Reliability for Cognitive Medical Systems}

Beyond data security, the hospital information systems must be aware that the measurement values recorded by consumer IoT smart devices are captured by from healthcare's point of view uncertified sensors. Therefore, these values must be flagged with the estimated quality, and the estimation calculation must be completed before these are fed into the corresponding industrial medical system [29]. Alternatively, the healthcare systems evaluate the calculated measurement quality and the values themselves accordingly.

The OTI hub flags every health-related data with the estimated quality. It stores the accompanying metadata linked to the health record, e.g. the type of the used sensor, sample rate, measurement error rate and delta comparison against the last measurement cycle. The OTI hub provides the metadata to the measurement values according to the specification in the data request.

The OTI hub also provides measurement statistics, e.g. real-time average. It assists data series visualization for the patient and general practitioner. The statistics service increases the measurement values' level of reliability; however, it also hides key values unveiling special disease types. The OTI hub supports parametrization for statistics services. The following expression [Figure 10] defines the applied real-time (moving) heart-rate average calculation in the OTI hub:

$$
\bar{v}_{S M}=\frac{v_{M}+v_{M-1}+v_{M-2}+\cdots+v_{M-(n-1)}}{n}=\frac{1}{n} \sum_{i=0}^{n-1} v_{M-(n-1)}
$$

Figure 10

The real-time heart-rate calculation formula

where $n$ is the number of the values in the series and $M$ is the total number of healthcare records.

The OTI hub calculates real-time statistics for the captured health-related time series. The statistics are provided beside the row values when the requesting application asks for them. These calculated statistics values are not stored in the Hub, but these are (re)calculated upon request.

\section{Conclusions}

Our proposed hybrid cloud architecture assures the essential scalability for the OTI-Hub in order to bear with the necessarily robust transaction processing capacity. The illustrated architectural topology and systems integration provides a technological solution for the integration of bi-directional international bodysensory, telemedicine, and classical healthcare data exchange. 
We learned from the experiment that the biggest challenge is the integration of the different data structures emitted by e-Health smart devices produced by alternative manufacturers. The illustrated results offer some optimism; however, current national healthcare data-related legal prerequisites need international harmonization to reach the required breakthrough. The illustrated OTI-Hub solution provides international e-Health data-exchange.

The IoT revolution dictates that industrial healthcare systems will deal with homeuse consumer smart devices equipped with multiple sensors. They are operating multiple types of bio-sensors and provide health-related bio-sensory raw data. These wearable devices provide valuable real-time information regarding their user and their environment. The collected information requires data cleaning and transformation.

These two steps signify the broken link in the integration of smart IoT devices in the overall healthcare supply chain. This is the reason, why the IoT technology still could not revolutionize the healthcare services domain. The next generation IoT bio-sensory sensors promise increased reliability and accuracy. When their precision reaches the critical threshold, then the spread of wearable IoT healthcare devices will be unstoppable.

The second success factor will be the free share and circulation of primary healthcare information. As people volunteer to share their medical raw information unanimously just easy as clicking on the pop-up menu at their smartphone app, new types of population-level disease follow-up and intervention will come into reality. This will also open new horizons for the human medicine.

\section{References}

[1] Huang Y., Kammerdiner A. Reduction of service time variation in patient visit groups using decision tree method for an effective scheduling, International Journal of Healthcare Technology and Management, Vol. 14, No. 1-2, 2013, pp. 3-21

[2] Kartsakli E., Antonopoulos A., Alonso L., Verikoukis C. A cloud-assisted random linear coding medium access control protocol for healthcare applications, Sensors, Special Issue on 'Sensors Data Fusion for Healthcare', 2014, pp. 9628-9668

[3] Baranyi P., Csapo A., Sallai Gy. Cognitive Infocommunications (CogInfoCom), Springer, 2015

[4] Baranyi P., Csapó Á. Definition and Synergies of Cognitive Infocommunications, Acta Polytechnica Hungarica, Vol. 9, No. 1, 2012

[5] Carlisle, James H. (June 1976) "Evaluating the impact of office automation on top management communication". Proceedings of the June 7-10, 1976, National Computer Conference and Exposition. pp. 611-616

[6] www.coginfocom.hu (last visited on 10.6.2018) 
[7] Izsó L. The significance of cognitive infocommunications in developing assistive technologies for people with non-standard cognitive characteristics: CogInfoCom for people with nonstandard cognitive characteristics, in Cognitive Infocommunications (CogInfoCom), $6^{\text {th }}$ IEEE International Conference on, Győr, 2015

[8] Vagner A. Cognitive Infocommunication for Monitoring and Improving Well-being of People, $8^{\text {th }}$ IEEE International Conference on Cognitive Infocommunications, Debrecen, 2017

[9] Magnusdottir E. H., Johannsdottir K. R, Bean C., Olafsson B., Gudnason J. Cognitive workload classification using cardiovascular measures and dynamic features, $8^{\text {th }}$ IEEE International Conference on Cognitive Infocommunications, Debrecen, 2017

[10] Marciniak R. Role of new IT solutions in the future of shared service model. Pollack Periodica, Vol. 8, No. 2, 2013, pp. 187-194

[11] Matusitz, Jonathan, \& Breen, Gerald Mark (2007) Telemedicine: Its Effects on Health Communication. Health Communication, 21(1), 73-83

[12] Bashshur R. L., Shannon G. W. History of telemedicine: Evolution, context, and transformation, New Rochelle, NY, Mary Ann Liebert, 2009

[13] Fong B., Fong A. C. M., Li C. K. Telemedicine technologies: Information technologies in medicine and telehealth, Chichester, Wiley, 2011

[14] Neelakantan P., Reddy A. R. M. Decentralized load balancing in distributed systems, Pollack Periodica, Vol. 9, No. 2, 2014, pp. 15-28

[15] Garai Á., Péntek I. Adaptive services with cloud architecture for telemedicine, $6^{\text {th }}$ IEEE Conference on Cognitive Infocommunications, Győr, Hungary, 19-21 October, 2015, pp. 369-374

[16] Adamkó A., Garai Á., Péntek I. Common open telemedicine hub and interface standard recommendation, The $10^{\text {th }}$ Jubilee Conference of $\mathrm{PhD}$ Students in Computer Science, Szeged, Hungary, 27-29 June, 2016, pp. 2425

[17] Adamkó A., Garai Á., Péntek I. Common open telemedicine hub and interface standard recommendation, The 10th Jubilee Conference of $\mathrm{PhD}$ Students in Computer Science, Szeged, Hungary, 27-29 June 2016, pp. 2425 [11] Adenuga O. A., Kekwaletswe R. M., Coleman A. eHealth integration and interoperability issues: towards a solution through enterprise architecture, Health Information Science and Systems, Vol. 3, No. 1, 2015, pp. 1-8

[18] ISO/IEC 7498-1, 1994 Information technology, Open systems interconnection, Basic reference model, The basic model (OSI-Model), International Organization for Standardization (ISO), Web, 6 June 2016 
[19] Garai L. Improving HPLC analysis of vitamin A and E: Use of statistical experimental design, International Conference on Computational Science, Zürich, Switzerland, 12-14 June, 2017, pp. 1500-1511

[20] Varshney U. Pervasive healthcare and wireless health monitoring, Mobile Networks and Applications, Vol. 12, No. 2, June 2007, pp. 113-127

[21] Martinez L., Gomez C. Telemedicine in the $21^{\text {st }}$ Century, Applied biostatistics for the health sciences, Nova Science Publishers, NY, 2008

[22] Poon C. Y., Hung K. F. mHealth: Intelligent closed-loop solutions for personalized healthcare, in Telehealth and mobile health, Eren H., Webster J. G. (Eds), CRC Press, 2015, pp. 145-160

[23] Garai L. Improving HPLC Analysis of Vitamin A and E: Use of Statistical Experimental Design, International Conference on Computational Science, Zürich, Switzerland, 12-14 June, 2017, pp. 1500-1511

[24] Zarour K (2016) Proposed technical architectural framework supporting heterogeneous applications in a hospital. International Journal of Electronic Healthcare 9:19-41

[25] Wootton R (1998) Telemedicine in the National Health Service. J. R. Soc. Med. 91: 289-292

[26] Wootton R (2012) Twenty years of telemedicine in chronic disease management an evidence synthesis. J. Telemed. Telecare. 18: 211220. doi: $10.1258 / \mathrm{jtt} .2012 .120219$

[27] Vigneshvar S, Sudhakumari C C, Senthilkumaran B, Prakash H (2016) Recent Advances in Biosensor Technology for Potential Applications An Overview Front. Bioeng. Biotechnol. 4:11. doi: 10.3389/fbioe.2016.00011

[28] Varshney, U (2007) Pervasive healthcare and wireless health monitoring. Mobile Networks and Applications 12: 2-3

[29] Rossi R J (2010) Applied Biostatistics for the Health Sciences. Wiley 